\title{
LIQUATION OF VYCOR GLASSES - STUDY BY POSITRON ANNIHILATION LIFETIME METHOD
}

\author{
B. JasińSKA ${ }^{a}$, A.L. DaWIDowicz ${ }^{b}$ AND S. RADKIEWICZ ${ }^{b}$ \\ ${ }^{a}$ Institute of Physics, Maria Curie-Skłodowska University \\ Pl. M. Curie-Skłodowskiej 1, 20-031 Lublin, Poland \\ ${ }^{b}$ Department of Chemical Physics, Faculty of Chemistry \\ Maria Curie-Skłodowska University \\ Pl. M. Curie-Skłodowskiej 3, 20-031 Lublin, Poland
}

Two Vycor glasses of different percentages of the components were liquated between $725 \mathrm{~K}$ and $950 \mathrm{~K}$ and leached in order to produce pores. Little intermolecular voids characterized by ortho-positronium lifetime value $\tau_{2} \approx 1.5$ ns were observed simultaneously. At the first stage of pore formation the intensity $I_{2}$ decreased considerably for both glasses, while at liquation temperatures higher than $825 \mathrm{~K}$ it increased again. The increase in $I_{2}$ resulted from changes in the properties of the bulk material. The growth of $\tau_{2}$ in this range of temperatures was also observed.

PACS numbers: 78.70.Bj, 61.43.Gt

\section{Introduction}

Positron annihilation lifetime spectroscopy (PALS) is commonly used in material science to investigate free volumes in various substances, particularly in polymers [1]. There exists a simple relation between ortho-positronium (o-Ps) lifetime and the average free volume size, assuming a spherical void shape [2, 3]. This relation is commonly used to determine free volume radii and their thermal expansion. In the case of solids the real shape of free space is, however, irregular; so, it seems to be quite reasonable to replace the existing model by, for example, a cuboidal one [4].

In the case of pores, their structure can be approximated as a bundle of capillaries. In standard measurements in physical chemistry, such as liquid nitrogen desorption, the average pore radius is defined as $R=2 V / S$, where $V$ and $S$ are the total pore volume and the surface area, respectively; that means that cylindrical geometry is assumed. It is then requisite to modify the Tao-Eldrup model for spheres to cylindrical geometry [5] and thus compare more easily the PALS results with those of conventional porosimetric methods. This modified model allows us to determine pore radii up to about $10 \mathrm{~nm}$.

The interpretation of $o$-Ps intensity is not always straightforward. Sometimes it can be interpreted as proportional to the number of free volume cavities [6], for 
example, in the case of low concentration of the admixture [7]. When more than one component ascribed to ${ }_{0}$-Ps can be extracted during the spectrum processing it is better to relate $o$-Ps intensity of the respective component to its surface area $[8,9]$.

\section{Experimental and material}

Two Vycor glasses of raw composition $7 / 23 / 70$ (the first one) and 10/35/55 per cent (the second one) $\mathrm{Na}_{2} \mathrm{O}, \mathrm{B}_{2} \mathrm{O}_{3}$, and $\mathrm{SiO}_{2}$ were investigated. Portions of glass grains were heated in an oven in order to demix the glass components. The extent of demixing depends on the temperature and duration of thermal treatment (liquation process). After liquation continuous alkaline-borate phase was formed in the silica network. Ten series of samples for different liquation temperatures between $725 \mathrm{~K}$ and $950 \mathrm{~K}$ in $25 \mathrm{~K}$ steps were prepared for each glass. For each annealing temperature, samples were prepared at different thermal treatment time. Glass samples were then etched in hot $(368 \mathrm{~K}) 3 \mathrm{~N} \mathrm{H}_{2} \mathrm{SO}_{4}$, alkaline-borate phase was removed out of the material and network of pores was consequently formed in the glass. Finally, the glass converted into porous sorbent was washed out to neutrality by means of distilled water and dried at $475 \mathrm{~K}$ for $12 \mathrm{~h}$.

All PALS measurements were performed at room temperature at a pressure of $\sim 0.5 \mathrm{~Pa}$.

For each glass sample PALS spectra were collected and then the lifetimes and intensities of respective components were determined. The spectrum was recorded in 4000 channels of an analyser, the channel time definition was $0.25 \mathrm{~ns}$. The time spectrum consisted of several components: the shortest-lived with $\tau_{1} \approx 0.45 \mathrm{~ns}$ was due to $p$-Ps decay and to free annihilation (not resolved), $\tau_{2} \approx 1.5 \mathrm{~ns}$ due to $o$-Ps annihilation in small interatomic voids in the glassy bulk and the longest-lived $\tau_{3} \approx 30 \mathrm{~ns}$ due to $o$-Ps in the pores. Respective relative intensities are denoted $I_{1}, I_{2}, I_{3}$. In the fitting procedure the LT [10] programme was used.

Before liquation and leaching both glasses were not porous, the lifetime of the longest-lived component was equal to $\tau_{2} \approx 1.32 \mathrm{~ns}$ and $1.44 \mathrm{~ns}$ for the $7 / 23 / 70$ and $10 / 35 / 55$ glasses, respectively. The intensity $I_{2}$ was about $32 \%$, which seems to be the saturation value for the $o-P s$ in the studied material. The third component, ascribed to pores, appeared after liquation. During spectrum processing a continuous distribution of lifetimes was assumed.

A detailed discussion of all processes observed during thermal treatment of $7 / 23 / 70$ Vycor glass was presented elsewhere [11]. In this paper we compare the processes observed during pore formation, leading to changes in the intensity $I_{2}$, in two glasses of different percentages of boron and sodium oxides.

\section{Results and discussion}

In both glasses subjected to liquation and leaching the pore network was produced. The average radius and structure of the pores changed with the liquation temperature. In the case of the first glass, the lifetime value of $o$-Ps component in the pores rose $6.5 \mathrm{~ns}$ to $40 \mathrm{~ns}$, in the second one from $13 \mathrm{~ns}$ to $50 \mathrm{~ns}$ [12]. This means that the pore radius determined assuming spherical shape of free volume $[2,3]$ rose 
from $0.56 \mathrm{~nm}$ to $1.33 \mathrm{~nm}$ or from 0.5 to $1.23 \mathrm{~nm}$ in the case of cylindrical shape [5]. For the second glass the spherical model gave values of radii changing from $0.77 \mathrm{~nm}$ to $1.57 \mathrm{~nm}$ while the cylindrical one from $0.7 \mathrm{~nm}$ to $1.57 \mathrm{~nm}$, too. The highest values of $\tau_{3}$ were observed for the $7 / 23 / 70$ glass liquated $16 \mathrm{~h}$ at $850 \mathrm{~K}$, and $16 \mathrm{~h}$ at $825 \mathrm{~K}$ for the $10 / 35 / 55$ one.
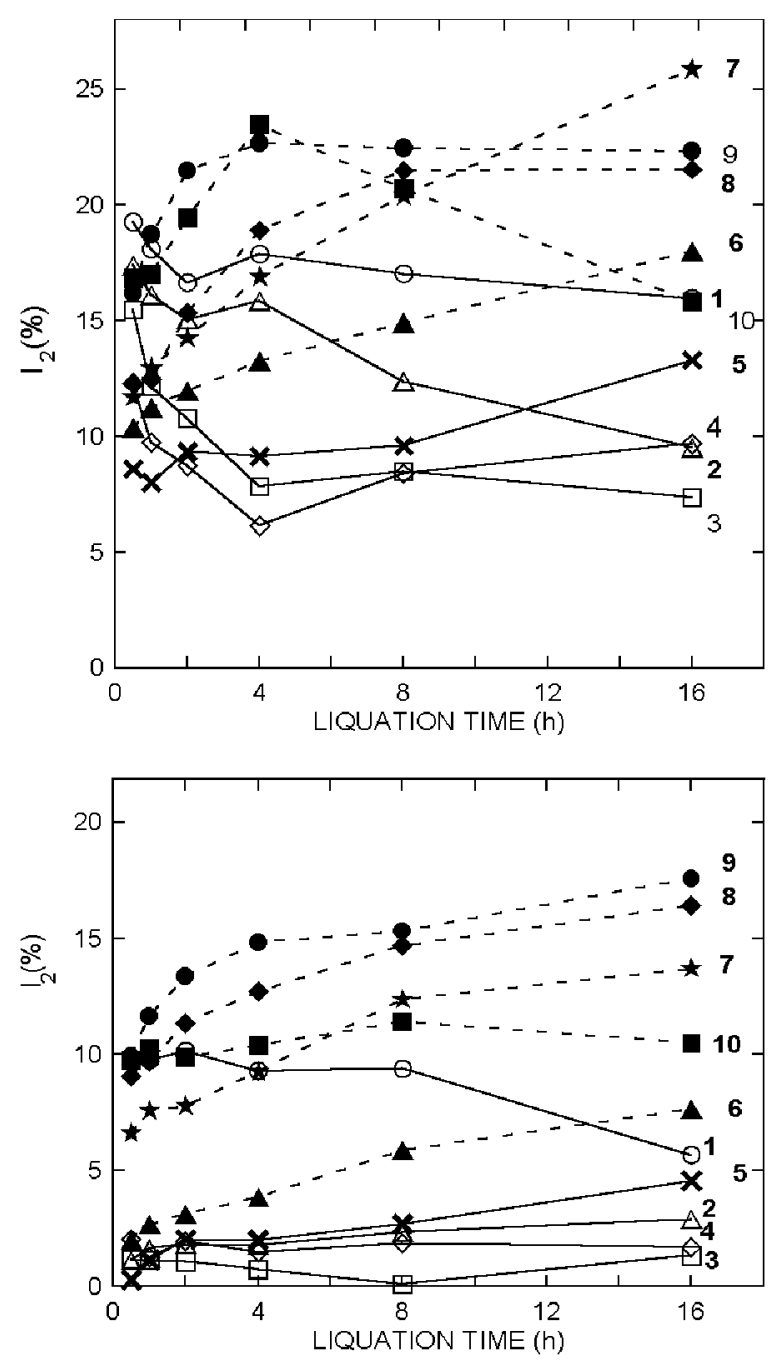

Fig. 1. The intensity $I_{2}$ of positronium in the intermolecular voids as a function of liquation time for 10 selected liquation temperatures. The upper figure is for $7 / 23 / 70$ per cent glass composition, the lower one for $10 / 35 / 55$. Symbols: open circles (1) $725 \mathrm{~K}$, open triangles $(2)-750 \mathrm{~K}$, open squares $(3)-775 \mathrm{~K}$, open diamonds (4) $800 \mathrm{~K}$, crosses $(5)-825 \mathrm{~K}$, full triangles $(6)-850 \mathrm{~K}$, stars $(7)-875 \mathrm{~K}$, full diamonds (8) $-900 \mathrm{~K}$, full circle $(9)-925 \mathrm{~K}$, full squares $(10)-950 \mathrm{~K}$. 

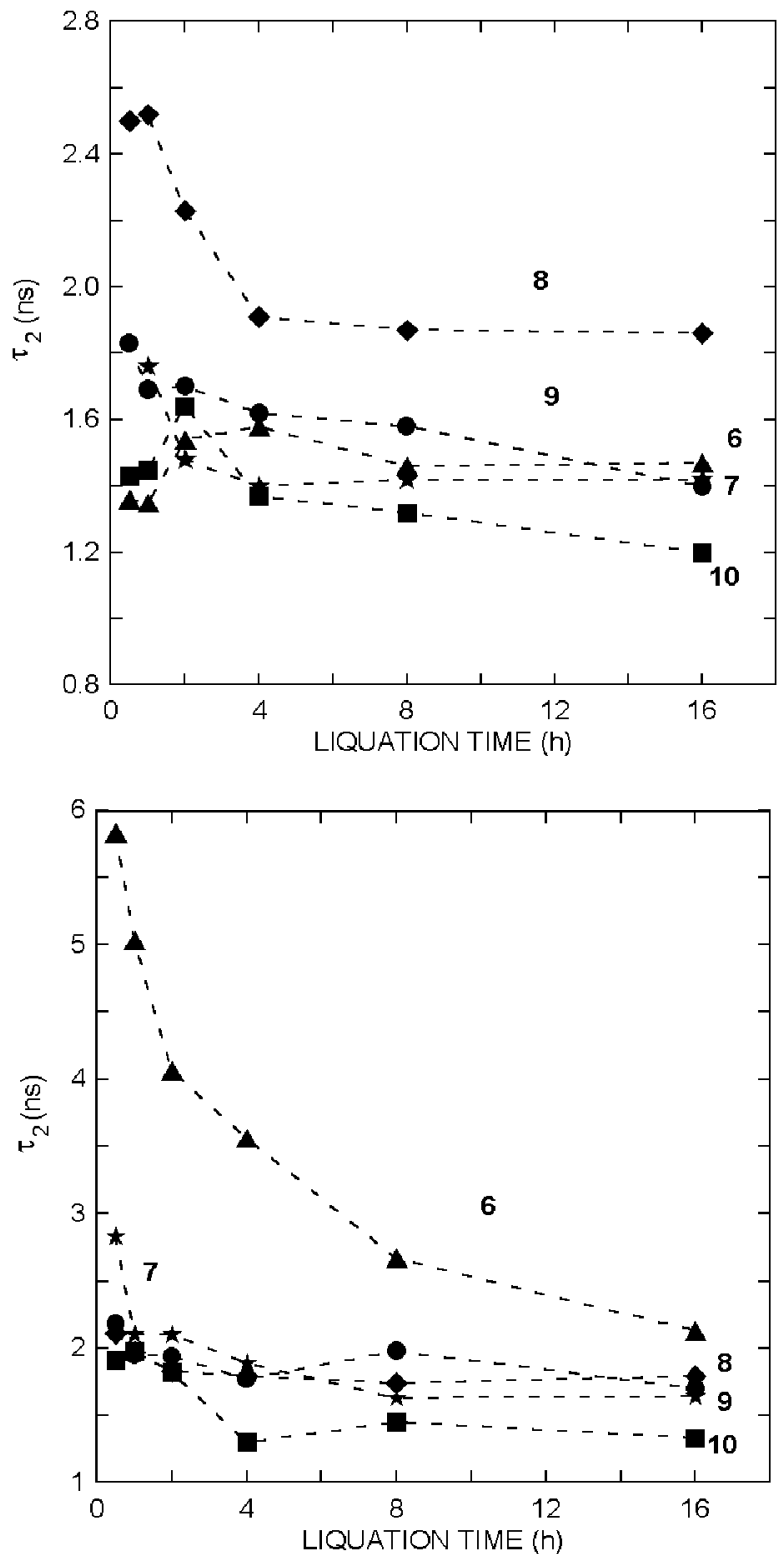

Fig. 2. The lifetime $\tau_{2}$ of positronium in the intermolecular voids as a function of liquation time for selected liquation temperatures. The upper figure is for $7 / 23 / 70$ per cent glass composition, the lower one for $10 / 35 / 55$. The curve numbers and symbols correspond to those in Fig. 1.

In the initial stage of pore formation, when the $I_{3}$ intensity began to grow, the second component intensity (with a lifetime of $1.32 \mathrm{~ns}$ or $1.44 \mathrm{~ns}$ ) decreased continuously (curves 1-3 in Fig. 1). The sum of both components reached still $32 \%$ 
as before liquation. According to the model by Brandt and Paulin [8], modified by Venkateswaran et al. [9], positronium initially locates in small intermolecular holes and then it diffuses to pores (and surfaces). The intensity of the bulk component diminishes with increasing pore surface due to Ps transitions to the pores. For the first glass $I_{2}$ fell down to about $7 \%$ and for the second one it almost disappeared in the temperature range of $750-825 \mathrm{~K}$ to the value of $I_{2} \approx 2 \%$ (Fig. 1, curves 2-5). Beginning from curve 6 the tendency was reversed for both glasses. Thus, one can suppose that after initial rapid pore formation, starting from $850 \mathrm{~K}$ on, the specific pore surface begins to decrease. The fraction of $o$-Ps atoms annihilating in the bulk $I_{2} /\left(I_{2}+I_{3}\right)$ can be approximated by the equation of Brandt and Paulin [8]

$$
I_{2} /\left(I_{2}+I_{3}\right)=1-\frac{3}{2} \beta\left[1-\beta^{2}+(1+\beta)^{2} \exp (-2 / \beta)\right]
$$

where $\beta=\frac{1}{3} S \rho\left(D \tau_{2}\right)^{1 / 2} ; S, \rho, D$ are the specific surface area of pores, the bulk glass density, and the Ps diffusion coefficient, respectively.

In a discussed range of temperatures this rate increases (as well as $I_{2}$ ), this means that a decrease of the pore surface is correlated with an increase of the intermolecular void surface. Changes in the o-Ps lifetime $\tau_{2}$ were also found (Fig. 2).

If in the spectrum analysis the lifetime of the second component is not fixed, its value is observed to increase to $2.5 \mathrm{~ns}$ at $900 \mathrm{~K}$ and $1.8 \mathrm{~ns}$ at $925 \mathrm{~K}$ for the $7 / 23 / 70$ glass; with a further increase in time or temperature of liquation, this lifetime value returns to the initial value, $1.3 \mathrm{~ns}$. For the $10 / 35 / 55$ glass the increase in the $\tau_{2}$ value is considerably higher: up to $\sim 6 \mathrm{~ns}$ at $850 \mathrm{~K}$. However, this value cannot be taken too seriously because of the low intensity of this component: $3-5 \%$ (curve 6 in Fig. 1), while the next one with the lifetime $\tau_{3} \sim 40$ ns has the intensity $I_{3} \sim 30 \%$. At low liquation temperatures a component ascribed to intermolecular voids can be hardly resolved, its intensity falls down below $2 \%$. However, in both glasses the rise of a lifetime value of this component is noticed.

Possible explanations for the observed lifetime variation are as follows:

1. Above the temperature specific for each glass composition, the glass shows liquid-like properties, so there is intense migration of glass components inside the grain. Molecules of sodium and borate oxides tending to inhomogeneities leave additional free space (which may be reflected in the $I_{2}$ growth), such voids are bigger than the natural ones in the glass (increase in $\tau_{2}$ ).

2. During thermal treatment at higher temperatures, a formation of a two-component alkaline phase inside the glass becomes possible (besides alkaline-borate also alkaline-silicate). During etching, the sodium-borate component is eluted and the sodium silicate compound decomposes into silicon dioxide, which as a secondary silica network is redeposited along the leached channel and creates a loose structure in which the distances between the settled molecules are bigger than in the bulk material, thus $\tau_{2}$ for o-Ps located in that structure is larger. With an increasing amount of silica the free spacings in the secondary structure are filled up. This mechanism of $\tau_{2}$ variations is less probable as Ps located very close to the pore surface would diffuse rapidly out to the bigger pore volume. 


\section{Conclusions}

The PALS method is commonly used in studies of materials for observations of little free volumes (a few angstroms size) in polymers, molecular crystals, liquids or for pore measurements (a few nanometers size). In our study both sizes of the voids were observed simultaneously. Pore radii were determined using a model assuming cylindrical shape of free volume. Using the data concerning intermolecular voids one can follow the processes occurring during thermal and chemical treatment of the material investigated. PALS seems to be especially useful to study the dynamics of processes proceeding in various substances.

\section{References}

[1] Y.C. Jean, in: Positron Spectroscopy of Solids, Eds. A. Dupasquier, A.P. Mills Jr., IOS Pub., Amsterdam 1995, p. 563.

[2] S.J. Tao, J. Chem. Phys. 56, 5499 (1979).

[3] M. Eldrup, D. Lightbody, J.N. Sherwood, Chem. Phys. 63, 51 (1981).

[4] B. Jasińska, A.E. Kozioł, T. Goworek, J. Radioanal. Nucl. Chem. 210, 617 (1996).

[5] K. Ciesielski, A.L. Dawidowicz, T. Goworek, B. Jasińska, J. Wawryszczuk, Chem. Phys. Lett. 289, 41 (1998).

[6] Y. Kobayashi, W. Zheng, E.F. Meyer, J. McGervey, A. Jamieson, Macromolecules 22, 2302 (1989).

[7] T. Goworek, C. Rybka, J. Wawryszczuk, Phys. Status Solidi B 89, 253 (1978).

[8] W. Brandt, R. Paulin, Phys. Rev. Lett. 21, 193 (1968).

[9] K. Venkateswaran, K.L. Cheng, Y.C. Jean, J. Phys. Chem. 88, 2465 (1984).

[10] J. Kansy, Nucl. Instrum. Methods A 374, 235 (1996).

[11] B. Jasińska, A.L. Dawidowicz, T. Goworek, Phys. Chem. Chem. Phys. 2, 3269 (2000).

[12] B. Jasińska, A.L. Dawidowicz, Mater. Sci. Forum, to be published. 